\title{
A speeded item response model with gradual process change
}

\author{
Yuri Goegebeur \\ Paul De Boeck \\ James A. Wollack \\ Allan S. Cohen
}

June 16, 2005

\begin{abstract}
An item response theory model for dealing with test speededness is proposed. The model consists of two random processes, a Rasch process and a random guessing process, with the random guessing gradually taking over from the Rasch process. The involved change point and change rate are considered random parameters in order to model examinee differences in both respects. The proposed model is evaluated on simulated data and a case study.
\end{abstract}

Key words: Rasch model, local item dependence, test speededness.

\section{Introduction}

Let $Y_{p i}$ denote the binary response (incorrect/correct, coded $Y_{p i}=0$ and $Y_{p i}=1$, respectively) of examinee $p, p=1, \ldots, P$, to item $i, i=1, \ldots, I$. In the classical one-parameter Rasch model (1PL) (Rasch, 1960) $Y_{p i}$ depends on the examinee ability $\theta_{p}$ and item difficulty $\beta_{i}$ in the following way

$$
Y_{p i} \mid \theta_{p} \sim \operatorname{Bern}\left(P_{i}\left(\theta_{p}\right)\right)
$$

with

$$
P_{i}\left(\theta_{p}\right)=\frac{\exp \left(\theta_{p}-\beta_{i}\right)}{1+\exp \left(\theta_{p}-\beta_{i}\right)}
$$

and $\theta_{p} \sim N\left(0, \sigma_{\theta}^{2}\right)$ if the marginal maximum likelihood formulation is chosen. Moreover, conditional on $\theta_{p}$ all responses of subject $p$ are assumed independent; this is the so-called local item independence condition. Formally, denoting $\mathbf{Y}_{p}^{\prime}=\left(Y_{p 1}, \ldots, Y_{p I}\right)$,

$$
P\left(\mathbf{Y}_{p}=\mathbf{y}_{p} \mid \theta_{p}\right)=\prod_{i=1}^{I}\left[P_{i}\left(\theta_{p}\right)\right]^{y_{p i}}\left[1-P_{i}\left(\theta_{p}\right)\right]^{1-y_{p i}} .
$$

The Rasch model has been extended in several ways. In the two-parameter logistic model (2PL) (Birnbaum, 1968) the difference $\theta_{p}-\beta_{i}$ is weighted by an item discrimination parameter $\alpha_{i}$ :

$$
P_{i}\left(\theta_{p}\right)=\frac{\exp \left(\alpha_{i}\left(\theta_{p}-\beta_{i}\right)\right)}{1+\exp \left(\alpha_{i}\left(\theta_{p}-\beta_{i}\right)\right)},
$$


so that the influence of examinee ability on outcome depends on the item. The three-parameter logistic model (3PL) (Birnbaum, 1968) extends the 2PL with an item specific guessing parameter $c_{i}$ :

$$
P_{i}\left(\theta_{p}\right)=c_{i}+\left(1-c_{i}\right) \frac{\exp \left(\alpha_{i}\left(\theta_{p}-\beta_{i}\right)\right)}{1+\exp \left(\alpha_{i}\left(\theta_{p}-\beta_{i}\right)\right)} .
$$

The parameter $c_{i}$ clearly reflects the probability of a correct answer under random guessing. For further interpretations of the 3PL, we refer to Hutchinson (1991).

The Rasch model, and item response theory models in general, are not robust with respect to violations of the local item independence assumption. The inclusion of items with local item dependence may result in contaminated estimates of test reliability, person and item parameters, standard errors and equating coefficients, see for instance Yen (1984), Thissen et al. (1989), Sireci et al. (1991), Yen (1993), Wainer and Thissen (1996) and Lee et al. (2001).

Yen (1993) and Ferrara et al. (1999) provide a detailed taxonomy of possible reasons for the existence of local item dependency. One of the most prevalent causes in educational testing is test speededness. Test speededness refers to testing situations in which some examinees do not have ample time to answer all questions. Speededness effects are often detrimental to the intended functioning of the test in that the speed with which one responds is usually not an important part of the construct of interest. Examinees affected by test speededness hurry through, randomly guess on or even fail to complete items, usually at the end of the test, and hence receive ability estimates that underestimate their capacities. On the other hand, the item difficulty parameters of items administered late in the test tend to be overestimated (Douglas et al., 1998 and Oshima, 1994).

Item response theory models dealing with test speededness are relatively new.

The hybrid model of Yamamoto and Everson (1997) uses multiple item response theory models to describe the behavior of examinees. A classical item response model is valid throughout most of the test but end-of-test items are answered randomly by some subset of examinees. The model identifies $M$ possible response patterns, one for whom an item response model is valid for all items, and $M-1$ patterns with an item response model describing answers to the first $I-m$ items and random guessing on the last $m$ items, $m=1, \ldots, M-1$. Formally,

$$
P_{i}^{(m)}\left(\theta_{p}^{(m)}\right)= \begin{cases}\frac{\exp \left(\alpha_{i}\left(\theta_{p}^{(m)}-\beta_{i}\right)\right)}{1+\exp \left(\alpha_{i}\left(\theta_{p}^{(m)}-\beta_{i}\right)\right)}, & i \leq I-m, \\ c_{i}, & i>I-m,\end{cases}
$$

with $m=0, \ldots, M-1$. Clearly, speededness is unlikely to be so straightforward, as students do not switch immediately to random guessing beyond some point.

Bolt et al. (2002) extend the mixture Rasch model proposed by Rost (1990) to distinguish latent classes of examinees according to the existence of speededness in their item response 
patterns. Ordinal constraints are imposed on the item difficulty parameters across classes so as to distinguish a class having no speededness effects from a class whose responses are affected by speededness. In particular, for items early in the test, the item difficulty parameters are constrained to be equal in the two classes; however, the item difficulty parameters of end-oftest items in the speeded class are constrained to be larger than the respective item difficulty parameters in the nonspeeded class. Let $g$ denote a class indicator with $g=0,1$ referring to the nonspeeded and speeded class respectively and let $k$ denote the first item where the examinees experience the effects of test speededness. The mixture Rasch model can then be stated as

$$
P_{i}^{(g)}\left(\theta_{p}^{(g)}\right)=\frac{\exp \left(\theta_{p}^{(g)}-\beta_{i}^{(g)}\right)}{1+\exp \left(\theta_{p}^{(g)}-\beta_{i}^{(g)}\right)},
$$

with

$$
\begin{array}{llll}
\beta_{i}^{(0)}=\beta_{i}^{(1)} & \text { for } & i<k \\
\beta_{i}^{(0)}<\beta_{i}^{(1)} & \text { for } & i \geq k .
\end{array}
$$

The item difficulty estimates obtained in the nonspeeded class provide more suitable estimates of the Rasch difficulties of end-of-test items than the difficulties estimated using all examinees. Although this model has worked quite well at identifying test speededness, it is likely overly simplistic as it does not allow for different examinees becoming speeded at different points in the test.

The remainder of this paper is organized as follows. In the next section we propose an item response model that accommodates the disadvantages of the hybrid model and the mixture Rasch model. The model can be seen as consisting of two random processes, a Rasch process and a random guessing process, with the random guessing gradually taking over from the Rasch process. The involved change point and change rate are considered random parameters in order to model examinee differences in both respects. The model was first formulated by Wollack and Cohen (2004) as a model to simulate speededness data, but it will be treated here as a full-fledged model for test data which can also be estimated. In section 3 we evaluate the performance of the model on the basis of a simulation study. The final section reports the results of applying the model to a mathematics placement test.

\section{A model for speeded test data with gradual process change}

In this section we propose a new item response model for dealing with speeded test data. Under the model, responses to items early in the test are governed by a Rasch model. Beyond some point the success probability gradually decreases and eventually reduces to the success probability under random guessing. Both change point and change rate are examinee specific.

Using the same notation as before, the model can be stated as

$$
Y_{p i} \mid \theta_{p}, \eta_{p}, \lambda_{p} \sim \operatorname{Bern}\left(\pi_{p i}\right)
$$


with

$$
\pi_{p i}=c_{i}+\left(1-c_{i}\right) P_{i}\left(\theta_{p}\right) \min \left\{1,\left[1-\left(\frac{i}{I}-\eta_{p}\right)\right]^{\lambda_{p}}\right\},
$$

where $P_{i}\left(\theta_{p}\right)$ is given by $(1)$ or $(2), \eta_{p}\left(\eta_{p} \in[0,1]\right)$ represents the speededness point and $\lambda_{p}$ $\left(\lambda_{p} \geq 0\right)$ the speededness rate of examinee $p$. The speededness point parameter $\eta_{p}$ identifies the point in the test, expressed as a fraction of the number of items, where examinee $p$ first experiences an effect due to speeding. For items with $i \leq \eta_{p} I$ there is no effect of speeding. Once the examinee passes his/her speededness point, $i / I-\eta_{p}$ is positive, resulting in a decrease of $\pi_{p i}$. The rate of decrease of $\pi_{p i}$ is controlled by the parameter $\lambda_{p}$, with larger $\lambda_{p}$ values resulting in a faster decrease. In Figure 1 we illustrate the role of $\eta$ and $\lambda$ by plotting the decay function $\min \left\{1,[1-(x-\eta)]^{\lambda}\right\}$ for some values of $\eta$ and $\lambda$.

(a)

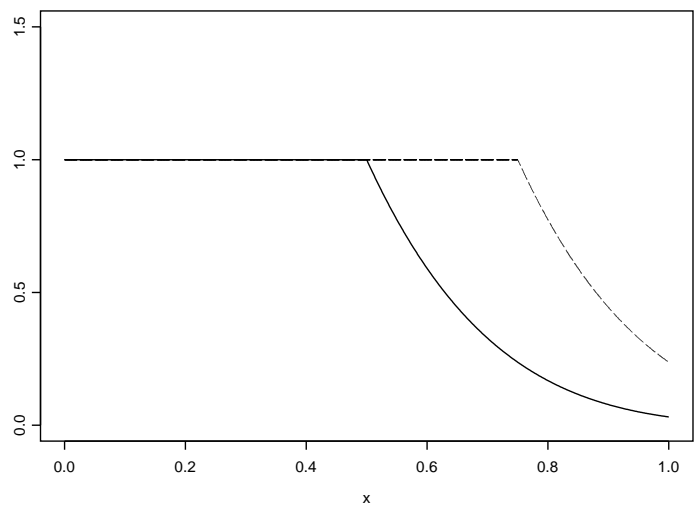

(b)

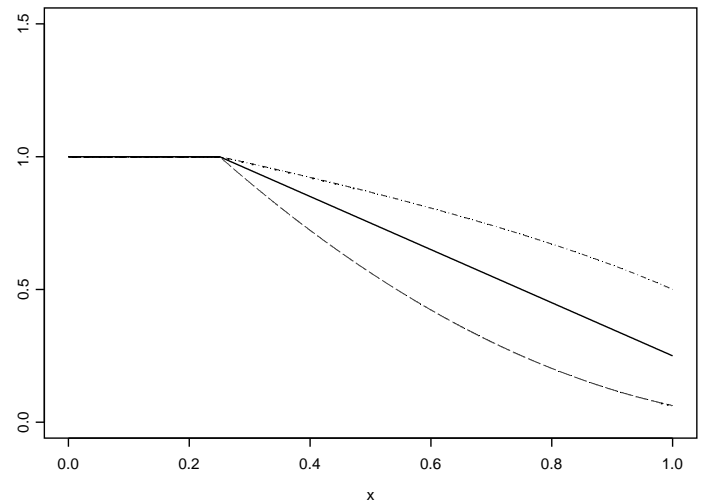

Figure 1: (a) $\min \left\{1,[1-(x-\eta)]^{\lambda}\right\}$ for $\lambda=5, \eta=0.5$ (solid line) and $\eta=0.75$ (broken line), (b) $\min \left\{1,[1-(x-\eta)]^{\lambda}\right\}$ for $\eta=0.25, \lambda=1$ (solid line), $\lambda=2$ (broken line) and $\lambda=0.5$ (broken-dotted line).

The rationale for the proposed model is as follows. Denote $P_{i}\left(\eta_{p}, \lambda_{p}\right)=\min \left\{1,\left[1-\left(i / I-\eta_{p}\right)\right]^{\lambda_{p}}\right\}$. When examinee $p$ encounters item $i$, he/she answers according to either a Rasch process or a random guessing process, with probabilities $P_{i}\left(\eta_{p}, \lambda_{p}\right)$ and $1-P_{i}\left(\eta_{p}, \lambda_{p}\right)$ respectively. Under random guessing the answer is correct with probability $c_{i}$. Under the Rasch process the examinee knows the answer with probability $P_{i}\left(\theta_{p}\right)$; if ignorant the examinee guesses at random. In Figure 2 we visualize the model with a decision tree. Clearly,

$$
P\left(Y_{p i}=1 \mid \theta_{p}, \eta_{p}, \lambda_{p}\right)=P_{i}\left(\eta_{p}, \lambda_{p}\right) P_{i}\left(\theta_{p}\right)+P_{i}\left(\eta_{p}, \lambda_{p}\right)\left[1-P_{i}\left(\theta_{p}\right)\right] c_{i}+\left[1-P_{i}\left(\eta_{p}, \lambda_{p}\right)\right] c_{i}
$$

which simplifies to (3).

Model (3) has some interesting limiting cases: 


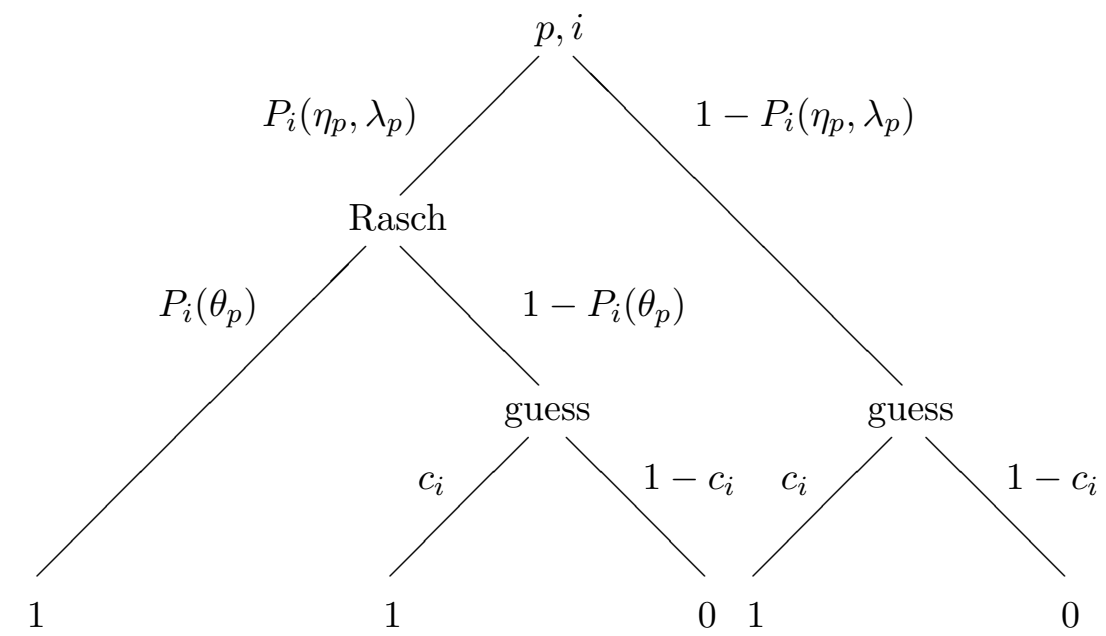

Figure 2: Decision tree representation of speededness model.

- if $[1-(i / I-\eta)]^{\lambda}=0$ for $i / I>\eta$ (this corresponds to the limiting case $\lambda \rightarrow+\infty$ ) then (3) reduces to one of the speeded classes in the hybrid model, and speededness is modeled as random guessing,

- in case $\lambda=0$ or $\eta=1$, the proposed model reduces to 1PL extended with random guessing or $3 \mathrm{PL}$,

- in case $\eta=0$ and $\lambda>0$ the examinee guesses at random at least to some degree for the first item up to the final item,

- similarly to $3 \mathrm{PL}, c_{i}$ is the lower asymptote for $\theta \rightarrow-\infty$.

As is usual in item response theory, the person ability parameter is assumed to be normally distributed with mean zero and variance $\sigma_{\theta}^{2}$. Concerning the parameters $\eta_{p}$ and $\lambda_{p}$ we make, without loss of generality, the following distributional assumptions:

$$
\begin{aligned}
\eta_{p} & \sim \operatorname{Beta}(\alpha, \beta), \\
\lambda_{p} & \sim \log N\left(\mu_{\lambda}, \sigma_{\lambda}^{2}\right) .
\end{aligned}
$$

For estimation we restrict the discussion to the marginal maximum likelihood method. If the model of interest is given by (3)-(1) with a common unknown random guessing parameter $c$, then the parameters to be estimated are $\left(\beta_{1}, \ldots, \beta_{I}, c, \sigma_{\theta}^{2}, \alpha, \beta, \mu_{\lambda}, \sigma_{\lambda}^{2}\right)$, whereas under (3)-(2) the parameters to be estimated are $\left(\alpha_{1}, \ldots, \alpha_{I}, \beta_{1}, \ldots, \beta_{I}, c, \alpha, \beta, \mu_{\lambda}, \sigma_{\lambda}^{2}\right)$. In the latter case $\sigma_{\theta}^{2}$ has to be fixed at some positive constant for identification purposes. For convenience the vector of unknown parameters will be denoted by $\boldsymbol{\xi}$. In the marginal maximum likelihood method the random effects are integrated out and the resulting likelihood is maximized with respect to be 
unknown parameters. Under (3) and denoting joint density function of $\theta_{p}, \eta_{p}$ and $\lambda_{p}$ by $g$ the marginal likelihood function is simply

$$
L(\boldsymbol{\xi})=\prod_{p=1}^{P} \int_{\mathbb{R}} \int_{0}^{1} \int_{0}^{\infty} \prod_{i=1}^{I} P\left(Y_{p i}=y_{p i} \mid \theta_{p}, \eta_{p}, \lambda_{p}\right) g\left(\theta_{p}, \eta_{p}, \lambda_{p}\right) d \lambda_{p} d \eta_{p} d \theta_{p} .
$$

The integrals involved in (4) can be numerically approximated by a quadrature method and the optimization can be performed using a standard Newton-Raphson algorithm. The SAS NLMIXED procedure fits nonlinear mixed models with multivariate normal random effect distributions. However, as long as $g$ in (4) is characterized by a normal dependence structure (copula) NLMIXED can be used to fit model (3), whatever the functional form of the (continuous) marginal random effect distribution functions. Indeed, as shown in Proposition 1 (see Appendix 2), in case of a normal dependence function, appropriately chosen compositions of probability integral transforms and inverse probability integral transforms of the marginal distributions yield a multivariate normal distribution for the transformed random effects. In some cases, besides $\boldsymbol{\xi}$ also the person specific effects $\theta_{p}, \eta_{p}$ and $\lambda_{p}$ are of special interest. Estimates of these parameters can be obtained from an empirical Bayes analysis of the postulated model.

\section{Simulation study}

In this section we discuss the results of a small simulation study. Three data sets each containing responses of 2000 examinees on 40 items were generated. Sample 1 was generated under model (3)-(1) with moderately high speededness $(\alpha=9$ and $\beta=2)$. Sample 2 was generated under model (3)-(1) with moderately low speededness $(\alpha=20$ and $\beta=9)$. Finally, a third sample was generated from 1PL with random guessing. The complete list of parameter values is given in Table 1. The random effects are assumed to be independent.

Table 1: Parameter values for simulation study.

\begin{tabular}{cccc}
\hline Parameter & Sample 1 & Sample 2 & Sample 3 \\
\hline$\beta_{1}-\beta_{40}$ & -1 & -1 & -1 \\
$c$ & 0.2 & 0.2 & 0.2 \\
$\sigma_{\theta}^{2}$ & 1 & 1 & 1 \\
$\alpha$ & 9 & 20 & - \\
$\beta$ & 2 & 2 & - \\
$\mu_{\lambda}$ & 0 & 0 & - \\
$\sigma_{\lambda}^{2}$ & 1 & 1 & - \\
\hline
\end{tabular}

The effect of test speededness is illustrated in Figure 3 (a), Figure 4 (a) and Figure 5 (a) where 
we plot the empirical proportions correct answers together with the theoretical ones, given by

$$
\begin{aligned}
\mathrm{E}\left(Y_{p i}\right) & =\mathrm{E}\left[\mathrm{E}\left(Y_{p i} \mid \theta_{p}, \eta_{p}, \lambda_{p}\right)\right] \\
& =\mathrm{E}\left(\pi_{p i}\right) \\
& =c+(1-c) \int_{\mathbb{R}} P_{i}\left(\theta_{p}\right) d G_{1}\left(\theta_{p}\right) \int_{0}^{1} \int_{0}^{\infty} \min \left\{1,\left[1-\left(\frac{i}{I}-\eta_{p}\right)\right]^{\lambda_{p}}\right\} d G_{3}\left(\lambda_{p}\right) d G_{2}\left(\eta_{p}\right),
\end{aligned}
$$

in case of (3) and by

$$
\mathrm{E}\left(Y_{p i}\right)=c+(1-c) \int_{\mathbb{R}} P_{i}\left(\theta_{p}\right) d G_{1}\left(\theta_{p}\right)
$$

in case of $1 \mathrm{PL}$ with random guessing, where $G_{1}, G_{2}$ and $G_{3}$ denote the distribution functions of $\theta_{p}, \eta_{p}$ and $\lambda_{p}$ respectively, versus item number. Since all $\beta_{i}$ are equal, these proportions should not depend on item number in the absence of test speededness (see Figure 5 (a)). Clearly, test speededness decreases the probability of a correct answer for end-of-test items. The ultimate effect depends on the distribution of the speededness point and rate.

To do:

- graphical presentation of estimation results

- test characteristic curves?? (cf Bolt, Cohen, Wollack)

\section{Application to mathematics placement test}

\section{Conclusion}

\section{Acknowledgements}

\section{References}

[1] Birnbaum, A., 1968. Some latent trait models and their use in inferring an examinee's ability. In Lord, F.M. and Novick, M.R. (Eds), Statistical Theories of Mental Test Scores, pp. 394-479. Addison-Wesly.

[2] Bolt, D.M., Cohen, A.S. and Wollack, J.A., 2002. Item parameter estimation under conditions of test speededness: application of a mixture Rasch model with ordinal constraints. Journal of Educational Measurement, 39, 331-348.

[3] De Boeck, P. and Wilson, M., 2004. Explanatory Item Response Models - A Generalized Linear and Nonlinear Approach. Springer.

[4] Douglas, J., Kim, H.R., Habing, B. and Gao, F., 1998. Investigating local dependence with conditional covariance functions. Journal of Educational and Behavioral Statistics, 23, 129151. 
[5] Ferrara, S., Huynh, H. and Michaels, H., 1999. Contextual explanations of local dependence in item clusters in a large-scale hands-on science performance assessment. Journal of Educational Measurement, 36, 119-140.

[6] Hutchinson, T.P., 1991. Ability, Partial Information, Guessing: Statistical Modelling Applied to Multiple-Choice Tests. Rumsby Scientific Publishing.

[7] Joe, H., 1997. Multivariate Models and Dependence Concepts. Chapman \& Hall.

[8] Lee, G., Kolen, M.J., Frisbie, D.A. and Ankenmann, R.D., 2001. Comparison of dichotomous and polytomous item response models in equating scores from tests composed of testlets. Applied Psychological Measurement, 25, 357-372.

[9] Nelsen, R.B., 1999. An Introduction to Copulas. Springer.

[10] Oshima, T.C., 1994. The effect of speededness on parameter estimation in item response theory. Journal of Educational Measurement, 31, 200-219.

[11] Rasch, G., 1960. Probabilistic Models for Some Intelligence and Attainment Tests. Danish Institute for Educational Research, Copenhagen, Denmark.

[12] Rost, J., 1990. Rasch models in latent classes: an integration of two approaches to item analysis. Applied Psychological Measurement, 14, 271-282.

[13] Sireci, S.G., Thissen, D. and Wainer, H., 1991. On the reliability of testlet-based tests. Journal of Educational Measurement, 28, 237-247.

[14] Sklar, A., 1959. Fonctions de repartition a $n$ dimensions et leurs marges. Publications de l'Institut de Statistique de l'Université de Paris, 8, 229-231.

[15] Thissen, D., Steinberg, L. and Mooney, J., 1989. Trace lines for testlets: a use of multiplecategorical response models. Journal of Educational Measurement, 26, 247-260.

[16] Wainer, H. and Thissen, D., 1996. How is reliability related to the quality of test scores? What is the effect of local dependence on reliability? Educational Measurement: Issues and Practice, 15, 22-29.

[17] Wollack, J.A. and Cohen, A.S., 2004. A model for simulating speeded test data. Technical report.

[18] Yamamoto, K. and Everson, H., 1997. Modeling the effects of test length and test time on parameter estimation using the hybrid model. In Rost, J. and Langeheine, R. (Eds.), Applications of Latent Trait and Latent Class Models in the Social Sciences, pp. 89-99. Waxmann, New York.

[19] Yen, W.M., 1984. Effects of local item dependence on the fit and equating performance of the three-parameter logistic model. Applied Psychological Measurement, 8, 125-145.

[20] Yen, W.M., 1993. Scaling performance assessments: strategies for managing local item dependence. Journal of Educational Measurement, 30, 187-213. 


\section{Appendix 1: Example SAS code}

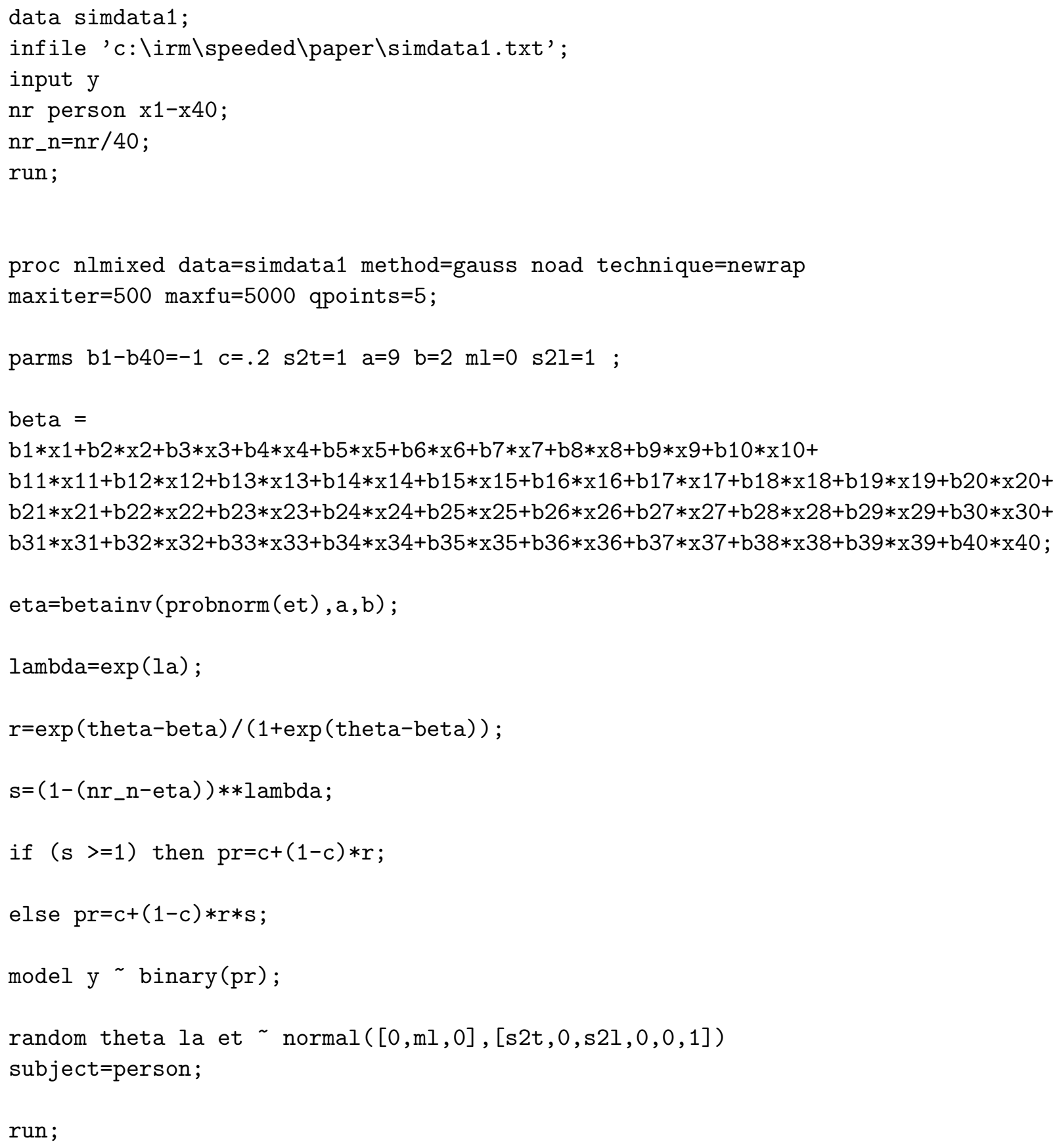




\section{Appendix 2}

Definition $1 A$-copula is a function $C:[0,1]^{n} \rightarrow[0,1]$ with the following properties

1. for every $\boldsymbol{u} \in[0,1]^{n}$ with at least one coordinate equal to $0, C(\boldsymbol{u})=0$,

2. if all coordinates of $\boldsymbol{u}$ are 1 except $u_{k}$ then $C(\boldsymbol{u})=u_{k}$,

3. for all $\boldsymbol{a}, \boldsymbol{b} \in[0,1]^{n}$ with $\boldsymbol{a} \leq \boldsymbol{b}$ the volume of the hyperrectangle with corners $\boldsymbol{a}$ and $\boldsymbol{b}$ is positive, i.e.

$$
\sum_{i_{1}=1}^{2} \cdots \sum_{i_{n}=1}^{2}(-1)^{i_{1}+\cdots+i_{n}} C\left(u_{i_{1}}, \ldots, u_{i_{n}}\right) \geq 0
$$

where $u_{i_{1}}=a_{i}$ and $u_{i_{2}}=b_{i}$.

So essentially a $n$-copula is a $n$-dimensional distribution function on $[0,1]^{n}$ with standard uniform marginal distributions. The next theorem, due to Sklar, is central to the theory of copulas and forms the basis of the applications of that theory to statistics.

Theorem 1 Sklar (1959) Let $\boldsymbol{X}^{\prime}=\left(X_{1}, \ldots, X_{n}\right)$ be a random vector with joint distribution function $F_{\boldsymbol{X}}$ and marginal distribution functions $F_{i}, i=1, \ldots, n$. Then there exists a copula $C$ such that for all $\boldsymbol{x} \in \mathbb{R}^{n}$

$$
F_{\boldsymbol{X}}(\boldsymbol{x})=C\left(F_{1}\left(x_{1}\right), \ldots, F_{n}\left(x_{n}\right)\right) .
$$

If $F_{1}, \ldots, F_{n}$ are all continuous then $C$ is unique, otherwise $C$ is uniquely determined on Ran $F_{1} \times \cdots \times$ Ran $F_{n}$. Conversely, given a copula $C$ and marginal distribution functions $F_{1}, \ldots, F_{n}$, the function $F_{\boldsymbol{X}}$ as defined by (5) is a joint distribution function with margins $F_{1}, \ldots, F_{n}$.

As is clear, Sklar's theorem separates a joint distribution into a part that describes the dependence structure (the copula) and parts that describe the marginal behavior (the marginal distributions). For further details on copula functions we refer to Joe (1997) and Nelsen (1999).

Proposition 1 Consider a n-dimensional random vector $\boldsymbol{X}$ with joint distribution function $G$ and continuous marginal distribution functions $G_{1}, \ldots, G_{n}$. Assume that $G$ is characterized by a normal dependence function (copula) $C$ i.e.

$$
G\left(x_{1}, \ldots, x_{n}\right)=C\left(G_{1}\left(x_{1}\right), \ldots, G_{n}\left(x_{n}\right)\right)
$$

with

$$
C\left(u_{1}, \ldots, u_{n}\right)=\int_{-\infty}^{\Phi^{-1}\left(u_{1}\right)} \cdots \int_{-\infty}^{\Phi^{-1}\left(u_{n}\right)} \frac{1}{(2 \pi)^{n / 2}|\boldsymbol{R}|^{1 / 2}} e^{-\frac{1}{2} \boldsymbol{z}^{\prime} \boldsymbol{R}^{-1} \boldsymbol{z}} d \boldsymbol{z}
$$

in which $\boldsymbol{R}$ denotes a (positive definite) correlation matrix and $\Phi^{-1}$ is the inverse standard normal distribution function. Then the random variables

$$
Y_{i}=\Phi^{-1}\left(G_{i}\left(X_{i}\right)\right), \quad i=1, \ldots, n,
$$

are jointly distributed as multivariate normal. 
Proof: Denote the joint distribution function of $Y_{1}, \ldots, Y_{n}$ by $H$. Then

$$
\begin{aligned}
H\left(y_{1}, \ldots, y_{n}\right) & =P\left(Y_{1} \leq y_{1}, \ldots, Y_{n} \leq y_{n}\right) \\
& =P\left(\Phi^{-1}\left(G_{1}\left(X_{1}\right)\right) \leq y_{1}, \ldots, \Phi^{-1}\left(G_{n}\left(X_{n}\right)\right) \leq y_{n}\right) \\
& =P\left(X_{1} \leq G_{1}^{-1}\left(\Phi\left(y_{1}\right)\right), \ldots, X_{n} \leq G_{n}^{-1}\left(\Phi\left(y_{n}\right)\right)\right) \\
& =C\left(\Phi\left(y_{1}\right), \ldots, \Phi\left(y_{n}\right)\right) \\
& =\int_{-\infty}^{y_{1}} \cdots \int_{-\infty}^{y_{n}} \frac{1}{(2 \pi)^{n / 2}|\boldsymbol{R}|^{1 / 2}} e^{-\frac{1}{2} \boldsymbol{z}^{\prime} \boldsymbol{R}^{-1} \boldsymbol{z}} d \boldsymbol{z},
\end{aligned}
$$

which is the distribution function of a multivariate normal distribution. 
(a)

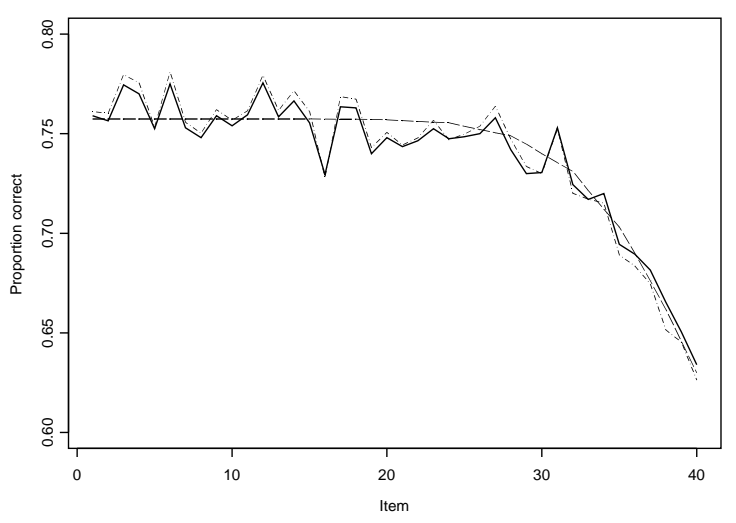

(c)

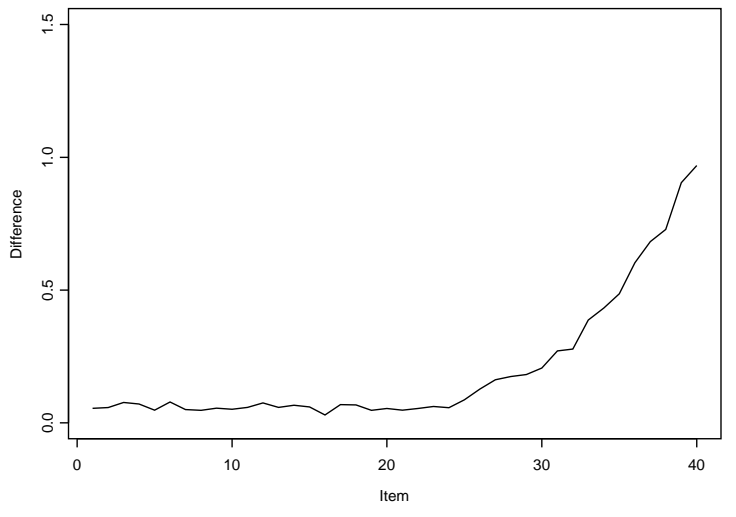

(e)

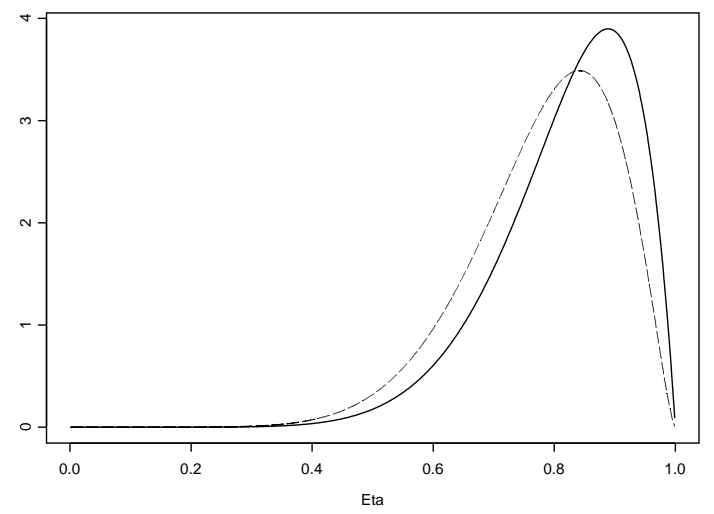

(b)

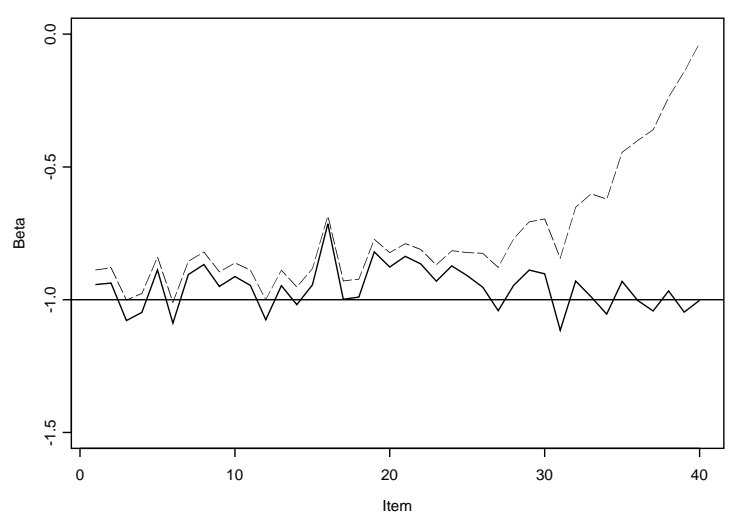

(d)

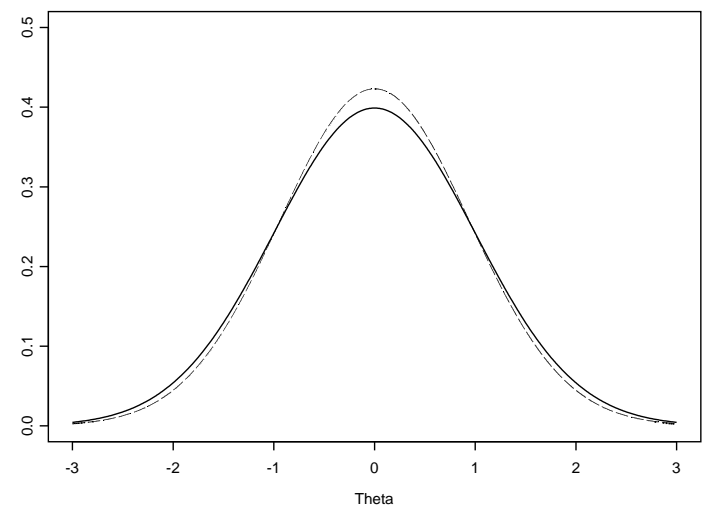

(f)

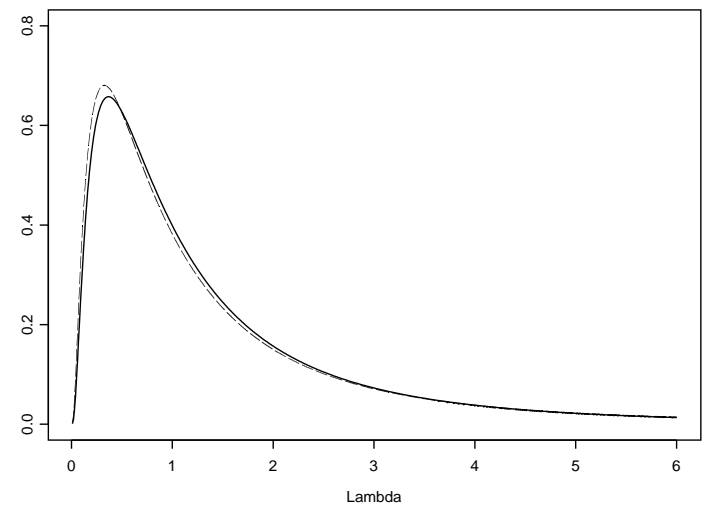

Figure 3: Results for sample 1 (a) proportion correct versus item number: empirical (solid line), theoretical with true parameter values (broken line), theoretical with estimated parameter values (broken-dotted line), (b) estimated item difficulty parameters under (3)-(1) (solid line) and 1PL with guessing (broken line), (c) difference between item difficulty estimates, (d) distribution of $\theta$ : theoretical (solid line) and fitted (broken line), (e) distribution of $\eta$ : theoretical (solid line) and fitted (broken line) and (f) distribution of $\lambda$ : theoretical (solid line) and fitted (broken line). 
(a)

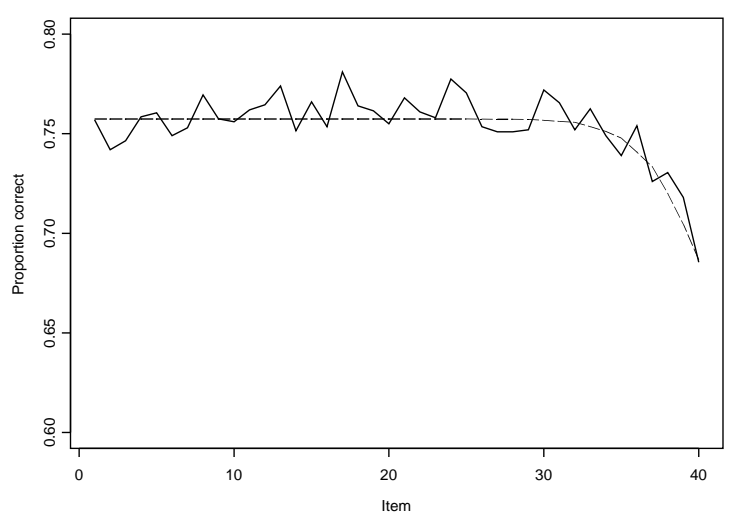

(e)

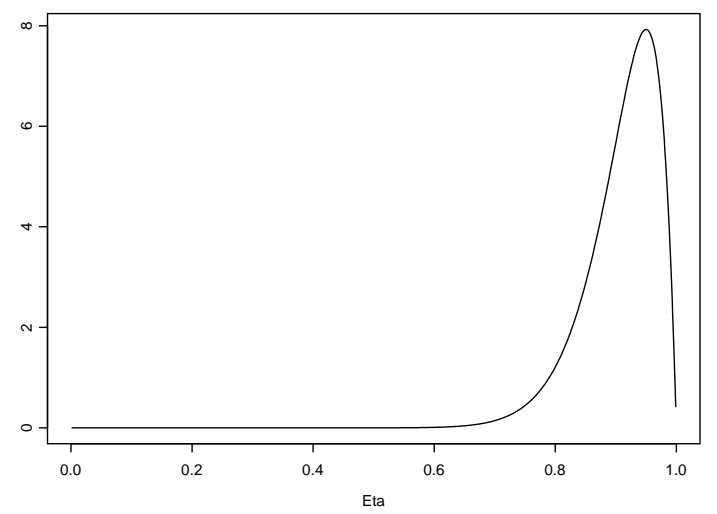

(d)

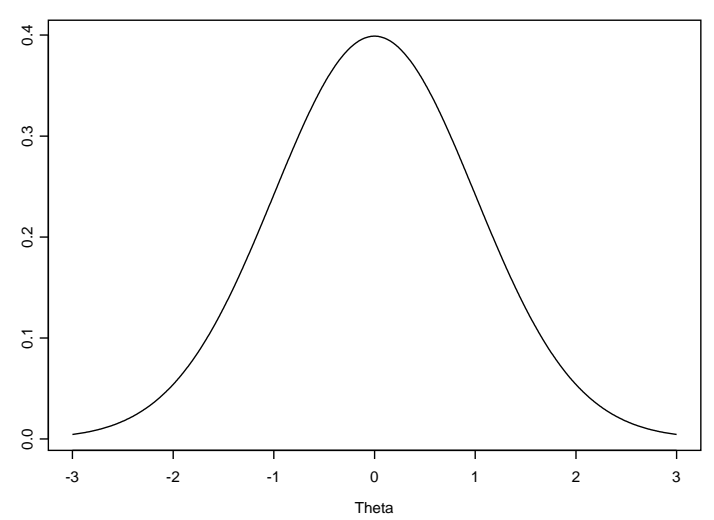

(f)

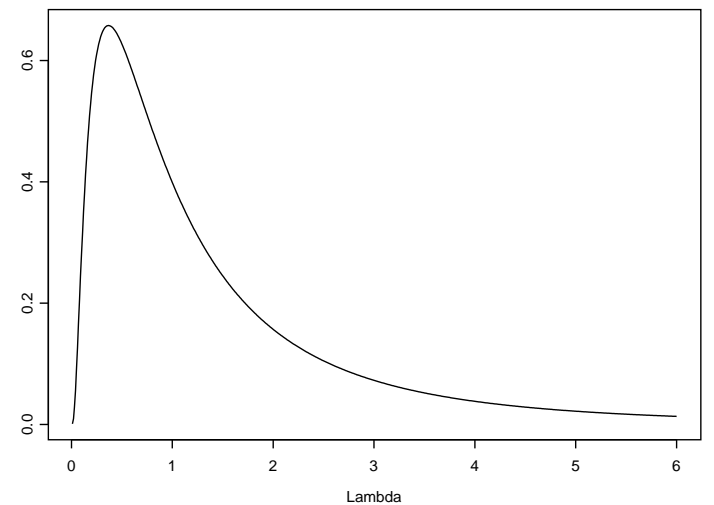

Figure 4: Results for sample 2 (a) proportion correct versus item number: empirical (solid line), theoretical with true parameter values (broken line), theoretical with estimated parameter values (broken-dotted line), (b) estimated item difficulty parameters under (3)-(1) (solid line) and 1PL with guessing (broken line), (c) difference between item difficulty estimates, (d) distribution of $\theta$ : theoretical (solid line) and fitted (broken line), (e) distribution of $\eta$ : theoretical (solid line) and fitted (broken line) and (f) distribution of $\lambda$ : theoretical (solid line) and fitted (broken line). 
(a)

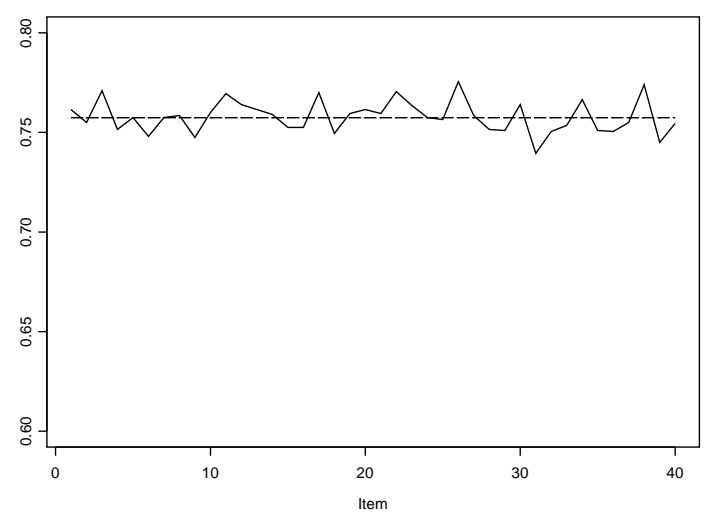

Figure 5: Results for sample 3 (a) proportion correct versus item number: empirical (solid line), theoretical with true parameter values (broken line), theoretical with estimated parameter values (broken-dotted line), (b) estimated item difficulty parameters under (3)-(1) (solid line) and 1PL with guessing (broken line), (c) difference between item difficulty estimates, (d) distribution of $\theta$ : theoretical (solid line) and fitted (broken line), (e) distribution of $\eta$ : theoretical (solid line) and fitted (broken line) and (f) distribution of $\lambda$ : theoretical (solid line) and fitted (broken line). 